\title{
Are Induction and Well-Ordering Equivalent?
}

\author{
Lars-Daniel Öhman $\odot$
}

(1) ver the course of the last few years, I have used in my teaching a variety of textbooks in the very broadly construed area of discrete mathematics. In one of these, the delightful Discrete Mathematics, by Norman L. Biggs, second edition [Big02], which I used in an introductory course in discrete mathematics, there is a section on how to introduce the natural numbers axiomatically, and in one subsection, the principle of mathematical induction is introduced as an axiom. This axiom is then used in a subsection on greatest and least members to prove that every nonempty subset of the natural numbers has a least member. The fact that the natural numbers have this property is usually called the well-ordering principle. It should be noted that sometimes this term is used for the well-ordering theorem, which states that every set can be well-ordered.

It seemed to me that there was something missing from the exposition, namely a remark along the lines that "we could have taken the well-ordering principle as an axiom instead of induction and still gotten the same structure, namely the familiar natural numbers." True enough, when I went back to the first (revised) edition of Biggs's book [Big89], which I had read for the introductory discrete mathematics course I took as an undergraduate, I found that the well-ordering principle was taken as an axiom, and the induction principle was a theorem. This would seem to indicate that either way of presenting things would yield the same outcome.

At this point, I decided to try to find a more solid, explicit, and detailed source for the equivalence of the induction principle and the well-ordering principle. So I searched for relevant articles in the MathSciNet database, and found the seemingly aptly titled article "The equivalence of the multiplication, pigeonhole, induction, and well-ordering principles" [HS88] and the related article "Placing the pigeonhole principle within the defining axioms of the integers" [HS80]. Unexpectedly, what piqued my interest was not the articles themselves, but rather the reviews by Perry Smith. According to the Mathematics Genealogy Project database, Smith is an academic grandson of the famous logician Stephen Kleene (whose doctoral dissertation, incidentally, is titled $A$ Theory of Positive Integers in Formal Logic), completing his PhD at UCLA in 1970 with a thesis entitled Some Contributions to Montague's Abstract Recursion Theory. Smith's publication record in MathSciNet comprises only two papers, but he has reviewed at least 169 papers in the database.

From the review of the article mentioned first, what I took away was the remark that "The authors work in Zermelo-Fraenkel set theory, but such arguments should be given in a weaker system of set theory or arithmetic in which the principles in question are not theorems" [Smi88]. The other review deserves to be cited in full:

The authors argue very informally that the pigeonhole principle can replace the induction axiom or the well-ordering principle in the set-theoretic characterization of the natural numbers. However, this claim must be formulated carefully if it is to be correct. For example, (1) the ordinals less than $\omega+\omega$ satisfy the first four (Dedekind-) Peano postulates and the well-ordering principle, but not the induction 
axiom or the pigeonhole principle; (2) the class of all cardinals satisfies the first four Peano postulates, the well-ordering principle, and the pigeonhole principle (if $m$ elements are distributed into $n$ boxes and $m>n$ then two elements must go into the same box), but not the induction axiom [Smi80].

I realized then that I had long suffered from a fundamental misconception. In the remainder of the present article, I will expand on the issue of in what exactly my misconception consisted, indicate that I was not alone in this misconception, and call on all good forces to help work against the further spread of it.

Regarding rigor, I do not aim to give a logically watertight presentation of the question at hand, but rather to supply a conceptual exposition of the ideas involved with enough details that trained mathematicians should feel able to bridge the technical gaps on their own, and that the interested amateur will at least be able to grasp what is at stake in these questions. In particular, I will not go into the question of first-order versus second-order versions of the induction axiom.

\section{A Fun Game to Illustrate What Is Going On}

Here's a game, the relevance of which will soon become clear, which I used to play with my three-year-old daughter. I would tell her a set of properties of a thing in the house, and she would guess which thing I was thinking of. As an example of an instance of the game, I would tell her that I'm thinking about something that

- has four legs,

- has a long tail,

- sleeps in her bed,

- is dark brown.

What am I thinking about? The answer, as she would tell me after a moment's thought, was her plush toy "Mousey." In mathematical terminology, we say that Mousey is a model for the set of clues, that is, a concrete example of something that satisfies all the properties. In fact, these four clues singled out a unique object-unique up to isomorphism, that is. Mousey was bought at Ikea, and has in fact been lost and replaced by an isomorphic copy on at least one occasion, unbeknownst to my daughter. In mathematical terminology, we say that these properties are categorical, that is, that they admit only one model.

It would then be her turn to give the clues, and often they would go something like this: I'm thinking about ...

- Daddy,

- who is sitting at the table,

- having breakfast.

The right answer, as I would figure out after a moment's thought, was indeed "Daddy."

In the following rounds of the game, I would vary the clues slightly, sometimes again singling out Mousey uniquely, and sometimes singling out uniquely another plush toy, "Tigris" (who, of course, is a tiger).
For instance, if I retained the first three clues, then the clue "is dark brown" could be supplanted with "has rodent teeth," and the set of clues would still single out Mousey. In mathematical terminology, we say that the clue "has rodent teeth" is equivalent to the clue "is dark brown" relative to the first three clues.

As another example, replacing "is dark brown" with "has sharp teeth" renders the set of clues no longer categorical, since both Tigris and Mousey are compatible with all of the clues. Therefore, the clues "is dark brown" and "has sharp teeth" are not equivalent relative to the first three clues. In mathematical terminology, we say that the set of clues has nonisomorphic models, the models being Mousey and Tigris.

On my daughter's turn to give clues, since "Daddy" usually remained the first clue, the question of which of the other clues were equivalent to each other given some base set of clues was less meaningful, since any true propositions about me would in some vacuous sense be equivalent to each other-they could replace each other freely, without affecting the categoricity of the set of properties. The only possible model would still be Daddy.

Peano, Induction, Well Ordering, and Equivalence In the late nineteenth century, Giuseppe Peano was thinking about a set $N$ together with a function $S: N \rightarrow N$ and a certain object 0 , with the properties that:

1. $0 \in N$, that is, 0 belongs to $N$;

2. If $n \in N$, then $S(n) \in N$;

3. $S(n) \neq 0$ for every $n \in N$;

4. If $S(n)=S(m)$, then $n=m$;

5. If $M$ is a subset of $N$ such that $0 \in M$ and $S(m) \in M$ for every $m \in M$, then $M=N$.

Please note that I do not intend to take a stand on the controversial question whether 0 is a natural number. None of the arguments in the present paper hinge on the inclusion of 0 among the natural numbers.

I have left out the axioms regulating how equality works, but it is reflexive, symmetric, and transitive, as would be expected. Note also that some concepts from set theory are assumed, at the very least the concept of set itself, the fundamental membership relation $\in$, and the equality of sets. The fifth property, or axiom, is the axiom of induction, or the induction principle.

Peano's function $S$ is usually called the successor function, and it conveys an order $<$ on the elements of $N$, by the following rules: for every $n \in N$, one has $n<S(n)$, and if $n<m$, then $n<S(m)$.

Now, as far as I can tell from biographical sources, Peano had no children, but if he had had children, he might have asked them what it was that he was thinking about. In light of Dedekind's proof that this set of axioms is categorical [Ded88], any guess other than (some isomorphic version of) the natural numbers, $\mathbb{N}$, would be wrong. In particular, guessing "the whole numbers" or "the ordinal numbers up to $\omega+\omega$ " would have been wrong.

Let us suppose that as the game went on, Peano would give the same first four clues, but instead of the fifth he would give the clue 
Every nonempty subset $M \subset N$ has a least member,

where the meaning of "least" is in relation to the order relation < defined on the basis of the function $S$. This is the well-ordering principle. Certainly, guessing "the natural numbers" could still be correct, since the natural numbers satisfy this property. However, guessing "the ordinal numbers up to $\omega+\omega$ " could not be refuted as an incorrect guess, since this model also satisfies properties (1)-(4) and $\left(5^{\prime}\right)$, as remarked by Perry.

Expanding on this remark, we denote the set of ordinal numbers up to $\omega+\omega$ by Ord. Here $\omega$ is the standard symbol used for the first limit ordinal, that is, the first ordinal to come after all the natural numbers. A numberline-style illustration of the ordinal numbers up to $\omega+\omega$ is given in Figure 1. The order relation $<$ on Ord is such that $n<\omega+m$ and $n<m+\omega=\omega$ for all natural numbers $n$ and $m$, and within each of the number lines, $<$ works as for the natural numbers. Note in particular that addition of ordinals is not commutative.

To see intuitively that every nonempty subset $M$ of Ord has a least member, suppose $M$ contains some ordinal numbers corresponding to ordinary natural numbers (that is, in the upper number line in the figure). Then the least member of $M$ is the least of these natural numbers. If $M$ contains only ordinals of the form $\omega+n$, then the least of them can be found by considering only the natural numbers in the $+n$ part of the ordinals in $M$. One could say that Ord inherits its well-ordering from the natural numbers, separately for each of the number lines in Figure 1.

In Ord, however, the induction axiom does not hold, since $\omega$ is not the successor of any of the previous ordinal numbers, and $\omega$ has no immediate predecessor.

In light of the above discussion, we draw two conclusions:

(A) The induction principle and the well-ordering principle are not equivalent relative to axioms (1)-(4) of the Peano system, since the resulting axiomatic systems admit different models.

(B) In the axiomatic system consisting of axioms (1)-(4) together with axiom $\left(5^{\prime}\right)$, induction (5) can't be a theorem, since there is a model Ord in which all these five axioms are satisfied, but induction (5) is not true.

We may also note that the axiomatic system consisting of axioms (1)-(5) admits only models that are isomorphic to the natural numbers, and since the natural numbers are well-ordered, in this system well-ordering $\left(5^{\prime}\right)$ is in fact a theorem. Induction (5) is therefore stronger than well-

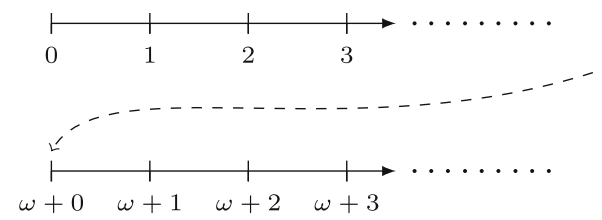

Figure I. Illustration of the set Ord, the ordinals up to $\omega+\omega$. ordering $\left(5^{\prime}\right)$ in this context, in that it has the power to rule out more possible models.

\section{Alternative Ways of Defining the Natural Numbers}

In this section, we look at a few alternative ways of formally introducing the natural numbers and the relationship between the well-ordering principle and the induction principle in these contexts.

\section{Set-Theoretic Definition}

To the human mind, the natural numbers may be a more primitive intuitive concept than sets, but that notwithstanding, the more common reduction in texts on the foundations of mathematics is usually that the concept of natural number is reduced to the concept of sets. In broad strokes, this goes as follows: Zermelo-Fraenkel set theory (with or without the axiom of choice) is introduced, including the axiom of infinity in particular, and the natural numbers are explicitly defined as, for example,

$$
0=\varnothing, \quad S(n)=n \cup\{n\} .
$$

Then the order relation $<$ on the integers corresponds to the inclusion relation $\in$ on sets, and both the well-ordering principle and the induction principle are theorems that hold for this structure, as remarked by Perry. In this context there is no sense in talking about whether the two properties are "equivalent." Instead, they are simply both true of the model.

\section{Algebraically Styled Definition}

Another, perhaps more intuitive, way of introducing the natural numbers is to lay down the rules governing the arithmetic operations + and $\times$ on the integers $\mathbb{Z}$. For instance, we then take as axioms that $a+b$ and $a \times b$ are integers whenever $a$ and $b$ are integers, that the left distributive law $a \times(b+c)=(a \times b)+(a \times c)$ holds for all integers $a, b, c$, and so on. We then add to this the rules governing the order relation, for example the transitive property, that $a \leq b$ and $b \leq c$ implies $a \leq c$. At this point, one can, in fact, define what a positive element is in the structure. That is, however, not yet enough to characterize the natural numbers. In particular, the set of rationals $\mathbb{Q}$ is still admissible as a model, so at least one more property is needed to pin down the natural numbers.

When introducing the natural numbers in this way, at this point, one may add either the well-ordering principle or the induction principle and actually get the same resulting uniquely characterized structure. In this context, then, the two principles are actually "equivalent," that is, they are equivalent relative to the other assumed properties. This version of formally introducing the natural numbers is the one used by Biggs [Big89, Big02].

\section{Special Case of General Ordinal Numbers}

Finally, I will just mention briefly the possibility of introducing the natural numbers as a special case of ordinal numbers. If this approach is employed, the order relation takes conceptual precedence to induction, and for general 
ordinals, the principle corresponding to "ordinary" induction is so-called transfinite induction. It is not straightforward to make sense of what exactly the question of the equivalence of the well-ordering principle and the induction principle should mean in this context. However, as with the set-theoretic definition, here both the wellordering principle and the induction principle hold.

\section{Looking at Textbooks}

Having convinced myself that induction and well-ordering are, in fact, not equivalent relative to the other Peano axioms, as described above, I actually updated my previously held belief. Unfortunately, I also had to update a remark in a textbook of which I myself was one of the authors [JO12]. This is an introductory textbook for firstsemester students of mathematics, and in the second edition, we no longer make the unspecific claim that the induction principle and the well-ordering principle are equivalent. As in some of the other sources I will now go on to cite, we were not explicit about which other defining properties of the natural numbers we had in mind, but I must admit that I was thinking about the Peano axioms.

I still felt that I should not have let myself be fooled, but as I checked the textbooks in various subjects I had come in contact with, either as a student or a teacher, I found remarks or claims to the same effect. I wish to state very clearly that I enjoyed all of these books greatly, and would recommend them to anyone. What follows here should therefore be taken for what it is: identifying isolated unfortunate formulations or inaccuracies in otherwise splendid sources, and an illustration of the range of sources who get this wrong, or at least misled me.

\section{Number Theory}

In number theory, I had taught a course using the textbook Introduction to Number Theory, by Erickson, Vazzana, and Garth [EVG16], where it is claimed that the principles are equivalent (p. 10), and it is left as an exercise to show this equivalence:

Show that [the] Principle of Mathematical Induction, Strong Mathematical Induction, and the Well Ordering Principle are all equivalent. That is, assuming any one holds, the other two hold as well (p. 11).

It is implicitly clear that this equivalence is intended to be relative to the other Peano axioms, since they are given in a supplementary section.

\section{Graph Theory}

In an undergraduate course in graph theory, I had read Introduction to Graph Theory, by West [Wes96], where (p. 15) the "Well Ordering Property" is assumed, and the principle of induction is proved as a theorem (see below for further details). It is unclear what other defining characteristics of the natural numbers are intended.

\section{Combinatorics}

In combinatorics, I had taught a course using the textbook Combinatorics-Topics, Techniques, Algorithms, by
Cameron [Cam94], where (pp. 10-11) the principle of induction is given, and the author goes on to "give some alternative forms of the Principle of Induction and justify their equivalence." Among these is proof by minimal counterexample, which is the well-ordering property in another guise: If a proposition $P(n)$ does not hold for all $n$, then there is some minimal $n$ for which it fails. However, the justification of equivalence is not actually given, but rather just an argument for why proof by minimal counterexample follows from the induction principle. Again, it is unclear what other defining characteristics of the natural numbers are intended.

\section{Algebra}

In abstract algebra, I had taught a course in which we recommend Abstract Algebra: Theory and Applications, by Judson [Jud18] as further reading. Here (p. 19), it is claimed that "The Principle of Well-Ordering is equivalent to the Principle of Mathematical Induction." However, only a (correct) proof that induction implies well-ordering is given. It is unclear, though, how the natural numbers are characterized.

\section{Specialized Texts on Induction}

In Handbook of Mathematical Induction-Theory and Applications, by Gunderson [Gun11], it is correctly proved that the standard order on the natural numbers as characterized by Peano's axioms is a well-ordering (p. 31), and transfinite induction is treated properly (pp. 53-54). However, despite this well-structured presentation, it is incorrectly claimed (p. 62) that induction follows from wellordering. The exposition is explicitly grounded in Peano's axioms

\section{What Goes Wrong?}

In the sources I have looked at that "prove" from axioms (1) - (4) and (5') that (5) holds, there is a common unjustified step of the proof, namely that every $n \in N$ has a unique immediate predecessor (perhaps denoted by $n-1)$. This property, however, does not follow from axioms (1)-(4) and $\left(5^{\prime}\right)$, as evidenced by the existence of a model Ord in which this property does not hold. Specifically, the limit ordinal $\omega$, for example, has no immediate predecessor. However, if the natural numbers are introduced in the more algebraic fashion described above, that step is justified.

The use of the immediate predecessor comes across clearly in [Gun11], where the following theorem and proof are given. Note that in the notation in the theorem, P5 corresponds to the induction principle and $x^{\prime}$ is the successor of $x$.

TheOREM 1 (WO $\rightarrow P 5$, [Gun11]). Assuming that the usual order on $\mathbb{N}$ is a well-ordering, then P5 holds.

Proof. Assume that $\mathbb{N}$ is well-ordered and assume that the hypothesis of $\mathrm{P} 5$ holds, namely that $S \neq \varnothing$ is a nonempty set of natural numbers with $1 \in S$ satisfying $(x \in S) \rightarrow\left(x^{\prime} \in S\right)$. Let $T=\{t \in S: t \notin \mathbb{N}\}$. To show that 
P5 holds, one must show that $S=\mathbb{N}$, that is, that $T=\varnothing$. In hopes of a contradiction, suppose that $T \neq \varnothing$. By well-ordering, $T$ contains a least element, say $t_{0} \in T$. Since $1 \in S$, it follows that $t_{0} \neq 1$. Since $t_{0}$ is the least element in $T$, one has $t_{0}-1 \notin T$, and so $t_{0}-1 \in \mathbb{N}$. By the hypothesis of $\mathrm{P} 5$, then $\left(t_{0}-1\right)^{\prime}=t_{0} \in \mathbb{N}$, contradicting that $t_{0} \in T$. So one must abandon the assumption that $T \neq \varnothing$ and conclude that $t=\varnothing$ and hence $S=\mathbb{N}$, thereby showing that P5 holds.

Note that the natural numbers were introduced by means of Peano's axioms, so if one removes the principle of induction from the axiom set and replaces it with the well-ordering property, the proof of Theorem 1 is incorrect.

Also note that the phrasing in the formulation of the theorem seems to suggest that we already have the natural numbers, and some ordering on them, but we do not yet know of this ordering that it is a well-ordering. As a second example of this, in [Wes96, pp. 10-11], we find the following theorem and proof, preceded by this remark:

The technique rests on the Well Ordering Property, which we ASSUME for the positive integers: every nonempty set of positive integers has a least element.

Theorem 2 (Induction principle [Wes96]). If $P(n)$ is a statement with an integer parameter $n$ and the following two conditions bold, then $P(n)$ is true for every positive $n$.

(1) $P(1)$ is true.

(2) For all $n \geq 1$, " $P(n)$ is true" implies " $P(n+1)$ is true."

Proof. Suppose that conditions (1) and (2) hold. If $P(n)$ does not hold for every positive integer $n$, then $P(n)$ fails for some nonempty set of positive integers. By the wellordering property, there is some least value for which $P(n)$ fails. By (1), this value cannot be 1 . By (2), this value cannot be any integer larger than 1 .

The proof does not explicitly use the immediate predecessor property, but it is implicit in the argument that "By (2), this value cannot be any integer larger than 1." Also, there is no mention of how exactly the natural numbers were actually introduced.

Saying that we assume the well-ordering property for the positive integers somehow indicates that we could imagine the positive integers as not having this property. Comparing this to the guessing game, this looks an awful lot like "I'm thinking about something that:

- is the positive integers;

- has the well-ordering property."

That is, saying that we assume the well-ordering property for the natural numbers is akin to saying that we assume that "Daddy" has some property that Daddy in fact has.

This raises the question of how the positive integers were characterized in the first place. By the Peano axioms?
By pure intuition? By giving a set-theoretic model for them in the Zermelo-Fraenkel axiom system for set theory? This question will be expanded upon below.

There thus seem to be three different misconceptions in play here. One higher-level misconception is the incorrect claim that axioms (1) - (4) together with $\left(5^{\prime}\right)$ characterize the natural numbers, via the induction principle, which is taken to be provable from these five axioms. The lower-level misconception that seems to underlie this is the incorrect assumption that the existence of (unique) successors together with the well-ordering principle implies the existence of (unique) predecessors. The third misconception seems to be more of a fallacy in reasoning, whereby the conclusion (a full characterization of the natural numbers) is assumed.

I will let Dedekind comment on the third point (as quoted in [Wan57]):

If one assumes knowledge of the sequence $N$ of natural numbers to begin with and accordingly permits himself an arithmetic terminology, then he has of course an easy time of it. He needs only to say: an element $n$ belongs to the sequence $N$ if and only if by starting with the element 1 , and going on counting, i.e. by a finite number of iterations of the mapping $k$ (compare the conclusion of 131 of my essay) I eventually reach the element $n$; on the other hand, I never reach an element $t$ outside the sequence $N$ by means of this process. But it is quite useless for our purpose to adopt this manner of distinguishing between those elements $t$ which are to be ejected from $S$, and those elements $n$ which alone are to remain in $S$. Such a procedure would surely involve the most pernicious and obvious kind of circulus vitiosus.

\section{Origins of the Misconceptions}

There are several different ways of asking about the origin of a phenomenon. Here, I shall briefly investigate only two aspects: how the phenomenon seems to have spread, and the first appearance of the components of the phenomenon.

\section{How the Misconceptions Have Spread}

It seems natural to assume that the wide diffusion of the imprecise claim that the induction principle and the wellordering principle are equivalent has been facilitated mainly by its inclusion in widely used textbooks. Specialized sources in axiomatics and set theory do not seem to make the mistake (see, for example [Sup60]). Rather, it is the sources that treat the axiomatic introduction of the natural numbers in a cursory fashion, as preliminaries to some other subject, that seem most likely to be sketchy on the details.

As mentioned above, I have myself been guilty of repeating this sketchiness in a textbook [JO12], so using introspection to analyze the mechanisms of how the misconception has spread indicates that doctrine (by which I mean knowledge spread through teaching) plays a central role. There are many textbooks, and I have unfortunately 
made only an incomplete effort to investigate influential textbooks from the period between the axiomatization of the natural numbers and the rise of mass education and a large market for textbooks.

My incomplete effort consists in investigating the two seemingly widespread books Naive Set Theory by Halmos [Hal60], and A Survey of Modern Algebra, by Birkhoff and Mac Lane [BML53].

In Naive Set Theory, the natural numbers (denoted by $\omega$ ) are introduced (p. 44) by means of the well-known explicit set-theoretic model in which $0=\varnothing$, and the successor is given by $x^{+}=x \cup\{x\}$. This is all well and good, and in Section 12, Peano's axioms are proved to hold. In Section 17, well-ordering is introduced together with transfinite induction, and it is cautioned that elements in well-ordered sets may fail to have predecessors. Despite these preparations, it is claimed that "the two statements are in general not equivalent to each other; their equivalence in $\omega$ is a happy but special circumstance" (p. 67). Here, therefore, we have an instance of claiming that the two theorems, induction principle and well-ordering principle, are equivalent for the natural numbers. Though it seems clear that Halmos was not confused about the relationship between the axioms, one may nonetheless imagine that this formulation may have contributed to confusion.

In A Survey of Modern Algebra, the integers are introduced by their desired algebraic properties and finally defined (p. 11) as an ordered integral domain in which the elements are well-ordered. The induction principle is then proved. Later (p. 54), the Peano axioms are given, and it is proved that they define a structure that is isomorphic to the natural numbers. In particular, it is proved that the successor function gives rise to a well-ordering. It is not explicitly stated that the well-ordering principle cannot replace the principle of induction among the Peano axioms. Again, one can therefore imagine how this treatment, while perfectly correct, may have contributed to confusion.

\section{First Appearances of the Components of the Misconception}

One way of actually making the well-ordering principle and the induction principle equivalent relative to the other axioms is to supplant axiom (3) with a slightly different version, namely:

$\left(3^{\prime}\right) \quad S(n) \neq 0$ for all $n \in N$, and 0 is the only element that is not a successor.

Axiom ( $\left.3^{\prime}\right)$ is obviously stronger than axiom (3). In a sense, this added strength makes up for the weakness of the wellordering principle in relation to the induction principle. Given the stronger axiom $\left(3^{\prime}\right)$, the two proofs above of the induction principle are correct. In fact, it seems that Dedekind considered using axiom (3') (see [Wan57]), but the version that made it into the standard Dedekind-Peano axioms was (3).

However, Mario Pieri, a contemporary of Peano, gave an alternative axiomatization of the natural numbers [Pie08] in which $\left(3^{\prime}\right)$ rather than (3) was used, and the well-ordering principle instead of the induction principle. He then proceeded to prove that his axiomatization and that of Peano were in fact equivalent. In [DLM01] it is suggested that confusion about the work of Pieri might be the root cause of the misconception that induction and well-ordering are equivalent in relation to axioms (1)-(4). However, it seems that Pieri's work on arithmetic did not receive much international attention at the time (see [MS07], p. 327), so the claim that Pieri's work is the root of the misconception does not seem convincing, even though it seems to mark the first appearance of the well-ordering principle in an axiomatic characterization of the natural numbers.

I hope that some historian of mathematics will take an interest in the question of the origins and spread of this unclarity.

\section{Applying the Principle of Charity}

When evaluating the arguments and claims of others, one should strive to apply a principle of charity, that is, to interpret those arguments and claims as charitably as possible. This would be the opposite of a so-called straw man argument, whereby one assigns untenable views to an opponent and proceeds to argue (successfully, of course) against those untenable views. In the current case, having identified the apparent misconceptions detailed above, one should then try to interpret them in a way that does not make them untenable or incorrect.

In looking at the actual incorrect deductions (using the Peano axioms) that well-ordering implies induction, I think it is hard to be charitable. In this system, using immediate predecessors is simply not warranted. I think, however, that there are two main points at which one can try to afford charity. First, perhaps something else is meant by "equivalence," for example in [Gun11], where "the equivalence between WO and P5" (p. 62) is discussed. Second, perhaps there is some reasonable interpretation of statements to the effect that some property is "assumed for the natural numbers."

\section{Equivalence Between Axioms}

Admittedly, the sources listed above are not primarily axiomatic treatments of the natural numbers, and so perhaps a more colloquial sense of the word "equivalence" is intended. That is, instead of claiming that the axioms (1) (5) single out the same model as the axioms (1)-(4) together with $\left(5^{\prime}\right)$ do, perhaps the intended sense is, for example, that the induction principle and the well-ordering principle are equally useful in proving theorems. Another possible intended meaning might be that for the natural numbers (maybe as given explicitly by some concrete model in set theory), both principles are equally true (that is, they are actually true, plain and simple).

If any of these other possible interpretations are what is actually intended, then I think that it would serve the reader better to state this in less misleading terms. I believe that the intuitive idea most mathematicians have, and that we should want to instill in students, is that the equivalence of two axioms is something relative to another base set of axioms. The two paradigmatic examples of this, which I 
believe shape mathematicians' understanding of equivalence of axioms, are the different versions of the parallel axiom in Euclidean geometry (equivalent relative to the rest of the standard axioms), and the axiom of choice versus, for instance, Zorn's lemma, in Zermelo-Fraenkel set theory. Therefore, regarding the statement that the well-ordering principle and the induction principle are "equivalent," I would think that the generally perceived meaning among readers is that they are equivalent relative to the other axioms, and I suspect that this is also usually the intended meaning on the part of the authors.

Taking Naive Set Theory as an example, it would seem that what is intended to be claimed is that the well-ordering principle and the induction principle are simultaneously true for the natural numbers, but that there are other structures (the ordinals, for example) in which they are not simultaneously true. Here, it could also be that what is intended is that transfinite induction in the case of the natural numbers coincides with ordinary induction.

\section{Properties "Assumed for the Natural Numbers"}

If it is assumed that the natural numbers are somehow at hand, by whatever mechanism, then "assuming" some property about them that is true for the natural numbers is certainly vacuous. Any arguments purporting to uncover some basic characterizing property for the natural numbers proceeding from this assumed property must then also be circular. This echoes the historical attempts to "prove the parallel axiom" in Euclidean geometry, where some "obvious" properties of the geometry were often tacitly assumed. In order to be charitable, one must therefore assume that what is intended by expressions such as "the well-ordering property, which we assume for the positive integers" is something like "assumed for this structure, which we are trying to pin down." In this case, there must still be some base set of axioms that is already in place.

As indicated above, using axiom $\left(3^{\prime}\right)$ instead of (3) actually makes the well-ordering principle equivalent to the induction principle, relative to the first four axioms. So perhaps this is what the authors quoted above actually had in mind. However, as remarked in [Gun11], "Peano's axioms are generally now accepted by the mathematical community as a starting point for arithmetic." In the sources I have seen that explicitly state the first four axioms, it is invariably axiom (3) that is used, not axiom $\left(3^{\prime}\right)$. This goes toward the argument that the mere existence of a base set of axioms relative to which (5) and $\left(5^{\prime}\right)$ are equivalent does not warrant the unspecified claim that they are equivalent.

In any case, formulations such as "assumed for the natural numbers" are questionable even in a slightly less formal treatment of the axiomatic grounds of numbers.

As noted above, I think it would be most interesting to see a more thorough historical investigation into these issues. Additionally, I have searched for, but not found, some source giving a more detailed overview of alternative ways of introducing and characterizing the natural numbers, perhaps also including an analysis of the relative strength of some different selections of axioms. This was investigated in a bachelor's thesis [SL16] that I supervised on the topic. An end goal would be for these issues to be as widely known as alternative axiomatizations of planar geometry and set theory.

\section{OPEN ACCESS}

This article is distributed under the terms of the Creative Commons Attribution 4.0 International License http://creativecommons.org/licenses/by/4.0/), which permits unrestricted use, distribution, and reproduction in any medium, provided you give appropriate credit to the original author(s) and the source, provide a link to the Creative Commons license, and indicate if changes were made.

Lars-Daniel Öhman

Department of Mathematics and Mathematical Statistics

Umeå University

90187 Umeå

Sweden

e-mail: lars-daniel.ohman@umu.se

\section{REFERENCES}

[Big89] Norman Linstead Biggs. Discrete Mathematics, revised ed. Oxford Science Publications, Oxford University Press, 1989.

[Big02] Norman Linstead Biggs. Discrete Mathematics, second ed. Oxford Science Publications, Oxford University Press, 2002.

[BML53] Garrett Birkhoff and Saunders Mac Lane. A Survey of Modern Algebra, revised ed. Macmillan, 1953.

[Cam94] Peter Jephson Cameron, Combinatorics: Topics, Techniques, Algorithms. Cambridge University Press, 1994.

[Ded88] Julius Wilhelm Richard Dedekind. Was sind und was sollen die Zahlen? Vieweg, 1888.

[DLM01] Maria Vittoria Di Leonardo and Teresa Marino. Is the least integer principle equivalent to the principle of mathematical induction? (il principio del minimo intero equivalente al principio di induzione completa?). Quad. Ric. Didatt. (2001), no. 10, 103114.

[EVG16] Martin J. Erickson, Anthony Matthew Vazzana, and David Ryan Garth. Introduction to Number Theory, second ed., Textbooks in Mathematics. CRC Press, 2016.

[Gun11] David S. Gunderson. Handbook of Mathematical Induction: Theory and Applications, Discrete Mathematics and Its Applications. CRC Press, 2011.

[Hal60] Paul Richard Halmos. Naive Set Theory, University Series in Undergraduate Mathematics. Van Nostrand, 1960.

[HS80] Rodney T. Hansen and Leonard George Swanson. Placing the pigeonhole principle within the defining axioms of the integers. In Proceedings of the West Coast Conference on Combinatorics, Graph Theory and Computing (Humboldt State Univ., Arcata, Calif., 1979), Congress. Numer., XXVI, Utilitas Math., Winnipeg, Man., 1980, pp. 183-186.

[HS88] Rodney T. Hansen and Leonard George Swanson. The equivalence of the multiplication, pigeonhole, induction, and well ordering principles. Internat. J. Math. Ed. Sci. Tech. 19:1 (1988), 129-131. 
[JO12] Robert Johansson and Lars-Daniel Öhman. Introduktion till högre studier i matematik. Liber Förlag, 2012.

[Jud18] Thomas William Judson. Abstract Algebra: Theory and Applications. Open source textbook available online at http:// abstract.ups.edu, 2018.

[MS07] Elena Anne Marchisotto and James T. Smith. The Legacy of Mario Pieri in Geometry and Arithmetic. Birkhäuser, 2007.

[Pie08] Mario Pieri. Sopra gli assiomi aritmetici. Bollettino dell'Accademia Gioenia di Scienze Naturali in Catania 2:2 (1908), 1-5.

[SL16] Malene Sandvær Lunnergård. "A refutation of the equivalence between the multiplication, pigeonhole, induction, and wellordering principles, with a main focus on the often claimed equivalence between the induction principle and the well-ordering principle when studying the natural numbers." Bachelor's thesis in mathematics, Dept. of Mathematics and Mathematical Statistics, Umeå University, 2016.
[Smi80] Perry Buckman Smith. Review of "Placing the pigeonhole principle within the defining axioms of the integers," by Rodney $T$. Hansen and Leonard G. Swanson. MathSciNet (1980).

[Smi88] Perry Buckman Smith. Review of "The equivalence of the multiplication, pigeonhole, induction, and well ordering principles," by Leonard G. Swanson and Rodney T. Hansen. MathSciNet (1988).

[Sup60] Patrick Colonel Suppes. Axiomatic Set Theory, University Series in Undergraduate Mathematics. Van Nostrand, 1960.

[Wan57] Hao Wang. The axiomatization of arithmetic. J. Symb. Logic 22 (1957), 145-158.

[Wes96] Douglas Brent West. Introduction to Graph Theory, Prentice Hall, 1996.

Publisher's Note Springer Nature remains neutral with regard to jurisdictional claims in published maps and institutional affiliations. 